\title{
Study Protocol: Developing a Culturally Appropriate Tobacco Control Project for the Vietnamese Community in South West Sydney, Australia
}

\author{
Khoi Dang Do, Raquiba Jahan Khan*, Sheree Whittaker, Karen Wardle, Natasha Poder \\ Health Promotion Service—-South Western Sydney Local Health District (SWSLHD), NSW Health, Liverpool, NSW, Australia.
}

How to cite this paper: Khoi Dang Do, Raquiba Jahan Khan, Sheree Whittaker, Karen Wardle, Natasha Poder. (2021) Study Protocol: Developing a Culturally Appropriate Tobacco Control Project for the Vietnamese Community in South West Sydney, Australia. Journal of Humanities, Arts and Social Science, 5(2), 225-230. DOI: $10.26855 /$ jhass.2021.07.006

Received: August 9, 2021

Accepted: August 31, 2021

Published: September 6, 2021

${ }^{*}$ Corresponding author: Raquiba Jahan Khan, Health Promotion Service-South Western Sydney Local Health District (SWSLHD), NSW Health, Liverpool, NSW, Australia.

Email: raquiba.khan@health.nsw.gov.au

\begin{abstract}
Introduction: The Vietnamese community in New South Wales (NSW) has been identified as a priority group for tailored anti-tobacco campaigns. Aim: Increasing quit attempts and reducing smoking-related harm in the Vietnamese community. Methods: Pre and post $(n=778)$ surveys will be used for evaluation. Randomly-selected telephone numbers of people with common Vietnamese surnames (aged $\geq 18$ years, residing in Fairfield) will be interviewed by telephone, using a questionnaire. The primary outcome of this study is the rate of quit attempts. An Epi Info database will be developed for data entry and the data will be analysed using SPSS Version-24. Three focus groups will be held with smokers, partners of smokers and ex-smokers recruited through baseline survey. These will explore barriers and enablers of using smoking cessation services; participants' awareness, knowledge, opinions of NSW Quitline, and the factors motivate or prevent participants accessing NSW Quitline. The data will be analysed thematically. Conclusion: The study has potential to contribute to the understanding of current tobacco use and engagement in quit support services in Vietnamese community. The study will provide insight in the processes used when developing tailored projects to address quit attempts in populations with high smoking rates.
\end{abstract}

Keywords

Smoking, Vietnamese, quit attempts

\section{Introduction}

Approximately seven million deaths each year are attributed to direct and second hand exposure to tobacco smoke (American Cancer Society, 2018; WHO, 2018). Tobacco smoking remains the leading preventable cause of death in Australia (Commonwealth of Australia, 2012; Scollo \& Winstanley, 2012).

In Australia, despite decreasing trends in smoking rates over the last 15 years (ABS, 2014-15), in some sub-population groups, the rates remain high (Cancer Institute NSW, 2013; Commonwealth of Australia, 2012; NSW Health, 2019). The Vietnamese community in New South Wales (NSW) has been identified as a priority group for tailored anti-tobacco campaigns (Scott et al., 2014). Daily tobacco use was higher among Vietnamese men compared to other CALD groups (Donato-Hunt, Munot, \& Copeland, 2012); and data show 32\% of Vietnamese men smoke, compared to the NSW state average for males of 14.7\% (NSW Health, 2010; HealthStats NSW, 2015).

The Vietnamese Tobacco Project (VTP), Bỏ Thuốc Sống Khỏe (Quit \& Fit), is a multi-strategic intervention to be 
undertaken by the South Western Sydney Local Health District's (SWSLHD) Health Promotion Service (HPS) in partnership with the South Western Sydney Primary Health Network (SWSPHN) and Fairfield City Council (FCC). The project team will work closely with the Cancer Institute NSW, who oversees the NSW Quitline which includes multilingual services. The intervention will be implemented in Fairfield Local Government Area (LGA) in Sydney, home to 39\% of the 102,896 Vietnamese-speaking residents of NSW (ABS, 2016). The project will be informed by previous experience gained through 'Health is Gold', an anti-smoking project for the Vietnamese community in south west Sydney (SWSAHS \& CSAHS, 1999).

\section{Methods\& design}

The project evaluation design will use quantitative and qualitative methods.

\subsection{Aim and Objectives}

The aim of the VTP is to increase quit attempts and reduce smoking-related harm in the Vietnamese community, Fairfield LGA, south west Sydney.

The project will assess self-reported smoking rates, rates of quit attempts and mean quit duration of smoking between baseline and follow up. Knowledge of smoking cessation support including the NSW Quitline will also be measured, alongside barriers and enablers to accessing these services.

\section{Quantitative Methods}

A cross-sectional study design with a pre-post survey method will be used to inform and evaluate the project strategies.

\subsection{Questionnaire}

A survey tool was developed based on surveys used in Australia and overseas. The survey was developed in English, and then translated into Vietnamese by a National Accreditation Authority for Translators and Interpreters accredited translator, before being checked by Vietnamese-speaking Health Promotion Officers (HPOs). The questionnaire was back-translated into English to check accuracy and content validity of the translation.

Four Vietnamese-speaking HPOs, including a staff member involved in survey development, analysis and interpretation, field-tested the questionnaire. The follow-up survey will include additional questions as appropriate on specific intervention messages and strategies.

The survey tool is divided into the following sections:

1. Smoke free environment (smoke free home and exposure to smoking).

2. Smoking behaviour (e.g. current smoking, cigarettes smoked per day, time to first cigarette).

3. Intention to quit, reasons to quit, confidence to quit.

4. Quit attempts and quit duration.

5. Use of products and type of support used for quit attempts.

6. Reasons for resuming smoking.

7. Attitudes to smoking.

8. Knowledge of smoking harms.

9. Perceptions of the effectiveness of smoking cessation support i.e. advice of a GP, using nicotine replacement and the NSW Quitline.

10. Demographics.

\subsection{Participants}

Participants' eligibility criteria includes: people with Vietnamese background; aged $\geq 18$ years; and live in a private residence in Fairfield LGA.

\subsection{Sampling}

The sample frame consists of 4,722 households with telephone numbers (landline and/or mobile) registered to common Vietnamese surnames in Fairfield LGA (Taylor, Nguyen, Do, Li, \& Yasui, 2011). Each household will be given an identification number and considered as one sample unit. The sample frame will be randomised and drawn from until the required sample size $(\mathrm{N}=778)$ is achieved. 


\subsection{Data collection}

Epi Info 7 will be used to create a computer-assisted telephone interview tool to guide interviewers through the process and control data-entry errors. Prior to commencing data collection, a training session will be organised by the research team. Training will include survey procedures and processes (e.g., how many times to call each household for making an appointment, choosing an eligible participant, explaining the study information sheet and consent process, automatic skipping and coding), telephone interview techniques and data entry using Epi Info 7. Staff will include a Vietnamese-speaking Bilingual Community Educator and HPOs (English and bilingual Vietnamese/English speaking staff).

Two weeks prior to initial phone contact, an information letter and participant information sheet, in Vietnamese and English, will be posted to selected households with a landline number. For participants with mobile numbers text messages will be sent, inviting them to take part in a phone survey.

For landline numbers, if there is more than one eligible person at the address, the person with the next birthday will be selected for interview. For mobile phone numbers the person who answers the phone will be invited to respond to the survey if the participant meets the eligibility criteria.

For each participant, up to five telephone calls will be made to make an appointment. If a participant cannot be contacted after five attempts, the next participant on the list will be contacted. Once scheduled, up to five attempts will be made to complete the phone survey.

\subsection{Recruitment for follow-up data collection}

During the baseline survey, participants will be invited to participate in the post survey. Participants who consent will be contacted when follow-up data collection commences. The remaining sample size will be randomly recruited using the same method as baseline.

\subsection{Data analysis}

The dataset will be reviewed for missing values, inconsistencies and outliers. The responses will be recoded where necessary. Data will be analysed using SPSS version 24. Coverage of the sampling frame will be estimated by comparing age and sex distribution of the survey sample with 2016 Census data for the Vietnamese community in Fairfield LGA. Weighted analysis will be performed if age and sex distribution in the survey differs significantly from Census data. Descriptive statistics (mean, standard deviation and frequency) will be performed to describe the participants' demographics.

The following inferential statistics will be performed to compare significant differences in the dependent variables of two or more groups (significance level: $P<0.05$ ) and to identify the factors associated with outcome variables:

- Associations between outcome variables (quit attempts, quitting duration, self-reported smoking status) and independent categorical variables (socio-demographic characteristics, attitude and knowledge) by using Pearson's chi-square test or Fisher's exact test.

- Pre- and post-intervention changes: T-tests will be used to compare the differences in the means of ratio/interval level variables before and after project implementation. In addition, one way analysis of variance(ANOVA) will be used where one independent categorical variable and one outcome ratio variable will be used to examine the significant differences in the mean scores in the ratio/interval variables (e.g. difference in the number of quit attempts by age or gender).

- Kruskal Wallis test will be used if data does not meet the parametric test assumptions. Factorial (two-way) ANOVA will be used to examine the effect of age and gender (two or more independent categorical variables) on the outcome variables such as mean quit attempt levels of males and females.

- $\quad$ Logistic regression models: For categorical outcome variables (smoking status and quit attempts), binary logistic models will be used to predict factors associated with the outcome of interest. For continuous outcomes (number of quit attempts and the length of abstinence after quitting), linear regression models will be used. For statistically significant main effects, pair-wise comparisons will be performed using Tukey's honesty significant difference test.

\section{Qualitative Methods}

AFGD tool has been developed to explore the barriers and enablers of using smoking cessation support services; assess participants' awareness, knowledge and opinions of NSW Quitline and the factors that either motivate or 
prevent participants accessing NSW Quitline. The follow up FGDs will further explore barriers to sustaining long term quit attempts. The FGDs will be held after completion of the baseline and follow up survey with smokers, partners of current smokers and ex-smokers recruited from the telephone survey. FGDs will take approximately one hour, facilitated in Vietnamese by two bilingual HPOs they will be electronically recorded and scribed. Prior to FGDs the facilitators will receive training from the research team about how to conduct a FGD.

\subsection{Analysis of FGD data}

The recorded data will be transcribed verbatim; each transcript will be reviewed for accuracy by the research team and bilingual Vietnamese speaking HPOs. The data will be analysed using a thematic analysis approach by two staff from the project including the Research Officer. The results will be presented to the team to confirm interpretation and for further elaboration.

\section{Project Interventions}

Findings from the baseline survey and focus group discussions will be used to guide strategy development, which will have a theoretical base in Social Learning Theory and the Stages of Change model. It is anticipated project strategies will include: encouraging use of evidence-based smoking cessation methods, including seeking advice from a GP or pharmacist; using NRT; accessing telephone support services to help support and maintain quit attempts. Other strategies that may be used include: community education sessions, healthy lifestyle programs, and conducting a creative arts competition to improve community awareness of smoking related harms. Key cultural events and festivals will also be used to maximise exposure and reach of project messages in the community. Project partners SWSPHN and FCC will also disseminate messages and support the engagement of health professionals, businesses and community members.

\section{Discussion}

There is considerable difference between some culturally and linguistically diverse (CALD) populations and mainstream smoking prevalence(Cancer Institute NSW, 2017; Scott et al., 2014; Cancer Institute NSW, 2013, NSW Health, 2019), despite the availability of evidence-based smoking cessation support services and more recently the provision of targeted in-language services. This research has the potential to contribute an understanding of current tobacco use, related behaviours and current engagement in quit support services in CALD communities, in particular the Vietnamese community. The research aims to identify current smoking behaviours, quit attempts, community attitudes and awareness of smoking related harm and cessation support. Findings will inform strategies to improve cultural appropriateness and acceptability of current services, increase utilisation of available smoking cessation support and engage the broader Vietnamese community to increase awareness and reduce the risk of smoking-related harm.

Routine population health surveys focus on collecting a representative sample of the state population. The numbers of CALD participants from the population health surveys may not be large enough for separate analyses. The benefit of this research is data collection with a large sample size from one CALD community. The mixed methods approach will enable the collection of detailed quantitative data including: cultural acceptability of accessing support; perceptions of evidence-based cessation methods and engaging with health professionals for advice alongside standard smoking and health questions. The focus groups will further enable exploration of the current patterns, and help to understand the cultural context, barriers and enablers to existing smoking cessation support options, while investigating the need for additional culturally appropriate services.

Involvement of the Vietnamese-speaking HPOs in the project planning and development is a strength of this intervention, their cultural expertise and established community networks will be leveraged to build trust and credibility within the Vietnamese community

\subsection{Limitations of the study}

Survey design excluded households with uncommon Vietnamese names, those without telephones or with unlisted/silent numbers. Mobile numbers will be purchased from a commercial provider. Participants who speak Vietnamese and do not have a common Vietnamese surname or no longer have an identifiable Vietnamese surname, such as those who have changed names due to marriage, may be missed from the sample. Despite this limitation using common surnames is a random sampling technique routinely used to select survey participants. 
Survey participants will be asked to self-report their current smoking status. Smoking status will not be validated. There is a possibility that survey respondents may under-report due to stigma or feeling ashamed of their smoking status. To minimise the sample bias, weighted analysis will be performed. Overall, the research will help generate evidence regarding the effectiveness of interventions designed to support and address smoking in CALD populations. The study will advance researchers' understanding of the subject area, inform future initiatives, and improve existing services and future priorities.

\section{Conclusion}

This study will contribute to the current evidence gap surrounding tobacco use in CALD populations, and in particular the understanding of smoking behaviours and access to evidence-based smoking cessation support in the Vietnamese community in south west Sydney. It will provide insight in the processes used and key considerations for health professionals when developing tailored projects to address quit attempts and reduced smoking-related harm in populations with high smoking rates.

\section{Ethical standards}

Ethics approval has been received from the SWSLHD Human Research and Ethics Committee Reference: HREC/16/LPOOL/489, Local Project Number: HE 16/258.Prior to starting the survey, the participant information statement (PIS) and the consent form will be read to the participants over the telephone, and they will be given an opportunity to ask questions before being asked for verbal consent. FGD participants will be given a FGD-specific PIS and a consent form to sign prior to commence the discussion session.

\section{Acknowledgements}

This project is funded by the Cancer Institute NSW through a Cancer Screening and Prevention Grant, E16/14057. We acknowledge the support provided by Fairfield City Council and South Western Sydney PHN as dedicated partners of the project. We would like to acknowledge the guidance, advice and support of Mandy Williams, Director - HPS/SWSLHD and Vilas Kovai HPS Research and Evaluation Manager in planning and developing the project. We thank our colleagues Amanda Eaton, Hien Le, Liliana Valenzuela-Flores and Lydia Farag for their work in literature review and needs assessment. We would also like to acknowledge the broader project team for their contributions and support. We would like to express our thanks to Vietnamese-speaking community members in Fairfield LGA for their participation in needs assessment.

\section{References}

ABS. (2014-15). ABS national health survey: first results 2014-15. Available at: http//www.abs.gov.au/AUSSTATS/ abs@.nsf/DetailsPage/4364.0.55.0012014-15?OpenDocument,accessed on 01/05/2019.

ABS. (2016) Census QuickStats. http://quickstats.censusdata.abs.gov.au/census-services/getproduct/census/2016/quick stat/LGA12850, accessed on 10 July 2018.

American Cancer Society. (2018). Vital strategies, The Tobacco Atlas 2018, sixth edition, the companion volume to the tobaccoatlas.org website - newly revised, expanded and updated. Available at: https://tobaccoatlas.org/wp-content/ uploads/2018/03/TobaccoAtlas_6thEdition_LoRes_Rev0318.pdf, accessed on $6^{\text {th }}$ Sept 2018.

Australian Institute of Health and Welfare. (2017). National Drug Strategy Household Survey 2016: detailed findings. Drug Statistics series no. 31. Cat. no. PHE 214. Canberra: AIHW.

Cancer Institute NSW. (2010). NSW Cancer Plan 2011-2015. Sydney.

Cancer Institute NSW. (2013). Tobacco smoking in Arabic, Chinese and Vietnamese communities in NSW: a qualitative exploration. Available at: http://uat-nsw.cancerinstitute.org.au/media/223758/report:cald.pdf., accessed on 13 July 2016.

Commonwealth of Australia. (2012). National tobacco strategy 2012-2018. Department of Health \& Ageing, Canberra, ACT, Australia.

Donato-Hunt, C., Munot, S., and Copeland, J. (2012). Alcohol, tobacco and illicit drug use among six culturally diverse communities in Sydney. Drug Alcohol Review, 31(7): 881-889.

HealthStats NSW. (2015). Current smoking in adults by local health districts, NSW. http://www.healthstats.nsw.gov.au/ Indicator/beh_smo_age/beh_smo_lhn (accessed on 13/7/2016). 
NSW Health. (2010). NSW Population Health Survey: 2006-2009 Report on adult health by country of birth. Available at:https://www.health.nsw.gov.au/surveys/adult/Publications/country-of-birth-06-09.pdf.

NSW Health. (2019). NSW Tobacco Strategy 2012-2021.

Scollo, M. and Winstanley, M. (2012). Tobacco in Australia: facts \& issues: Cancer Council Victoria; 2012.

Scott, N., Donato-Hunt, C., Crane, M., Lafontaine, M., Varlow, M., Seale, H., et al. (2014). Knowledge, attitudes and beliefs about lung cancer in three culturally and linguistically diverse communities living in Australia: a qualitative study. Health Promotion Journal of Australia, 25(1): 46-51.

SWSAHS and CSAHS. (1999). Evaluation of an anti-smoking project targeting Vietnamese men in south western Sydney: an unpublished report. Sydney.

Taylor, V. M., Nguyen, T. T., Do, H. H., Li, L., and Yasui, Y. (2011). Lessons learned from the application of a Vietnamese surname list for survey research. Journal of Immigrant and Minority Health, 13(2): 345-51.

WHO. (2018). Available at: http://www.who.int/news-room/fact-sheets/detail/tobacco, accessed on 6 Sept 2018. 